\title{
Assessing measurement uncertainty in CMM measurements: comparison of different approaches
}

\author{
S. Ruffa ${ }^{\star}$, G.D. Panciani, F. Ricci, and G. Vicario \\ Politecnico di Torino, Corso Duca degli Abruzzi 24, 10129 Torino, Italy
}

Received: 5 July 2013 / Accepted: 31 October 2013

\begin{abstract}
Manufactured parts are affected by size and form errors, which need to be assessed against geometrical and dimensional tolerances in order to meet the functional requirements they have been conceived for. The compliance assessment of workpieces with specifications depends on measurements and is unavoidably affected by several uncertainty contributions. According to the geometrical product specifications and verification (GPS), measurement uncertainty consists of method and implementation uncertainty. Literature proposes different approaches for the evaluation of implementation uncertainty, however a standardized method has not been yet achieved. This paper makes an analysis of the various elements to be considered when choosing a specific approach: in the implementation uncertainty of circular features. The most common manufacturing signatures affecting circular profiles were considered, together with the number of points necessary for a reliable estimation of implementation uncertainty.
\end{abstract}

Keywords: Uncertainty; geometrical product specification and verification; coordinate measuring machine; roundness; form error

\section{Introduction}

Manufactured parts are affected by form and size errors that have to be assessed against geometrical and dimensional tolerances in order to prove their conformance with specification. The geometrical product specification and verification (GPS) standards lay the groundwork for a new operator based language, able to manage a verification coherent with specifications and, therefore, to assure the consistency of information, together with the reduction of total uncertainty during the product lifecycle [1].

This paper deals with the verification of roundness specification in the light of GPS standards. Roundness tolerance is meant to control the form of features that are nominally circular. According to the GPS Masterplan, standards necessary for the correct interpretation of geometrical specifications are organized in chains of "general standards". There is one chain of general standards for each geometrical specification and each chain addresses in this order: specification, verification and instrument calibration issues. According to roundness tolerance, reference standards are the ISO 12181-1 [2] for points extraction, association and evaluation, and the ISO 12181-2 [3] for points filtration.

If all the information required by these standards is annotated in the tolerance callout, both specification and verification operators are defined: it is clearly defined what has to be controlled and how it shall be done. On the other hand, during verification, there are two alternatives: to replicate faithfully the specification procedure, defining

^ Correspondence: suela.ruffa@polito.it a perfect verification operator (actual verification operations), or perform different simplified verification operators, to choose the best trade off in terms of time and cost. The correct identification of the actual roundness profile is extremely important. Most roundness deviation is systematic apart from random error and may be interpreted as the signature of the manufacturing processes.

The next section is devoted to defining roundness specification (and verification) operators, as provided by ISO 12181-2. Section 3 describes the different uncertainty contributions, in compliance with GPS classification. Then, in Section 4, methods for computing implementation uncertainty are compared for the measurement of circular profiles. In particular, we simulate measurements on virtual circular profiles in order to avoid not knowing the actual shape of a workpiece before measuring it. Circular profiles have been simulated through Fourier transform, using harmonics that range from low frequency form errors to roughness, as it naturally highlights the correspondence between the spectra of fundamental harmonics and the possible errors of manufacturing processes [4]. A final discussion, on the basis of the experiments performed, compares results from different methods.

\section{Roundness specification and verification}

According to ISO 12181-1, roundness tolerance is defined as the zone between two concentric circles which enclose the circular profile. It limits the roundness deviations, that is the distance between two concentric circles touching and 
enclosing the extracted circle (i.e. measured points) at the minimum radial distance from each other.

The roundness complete specification operator, according to ISO 12181-2, settles all the operations necessary to verify the compliance of the actual feature with the designer's intent. All operations are briefly described below with respect to roundness verification using a coordinate measuring machine (CMM). This leads to a set of extracted points with coordinates $\left(x_{i}, y_{i}\right), 1 \leqslant i \leqslant n$.

In this paper, the coordinates of measurement points are obtained by simulating a touch probe inspection. Particularly the touch probe is a spherical tip stylus with radius depending on the measurand diameter and the nature of error to be assessed [5].

According to ISO 12181-2 a proper filter cut-off wavelength $\lambda_{c o}$ must be selected in order to smooth the effect of measurement errors and extract the component of form error required by the geometrical specification (e.g. to separate form error from waviness and roughness).

Moreover, the filter characteristic defines the minimum number of points to be sampled, since the original signal is bandwidth limited. According to Nyquist theorem, in order to avoid aliasing, at least seven points per undulation per revolution (UPR) must be sampled, where $U P R=2 \pi R / \lambda_{c o}$ and $R$ is the measurand radius.

ISO proposes different filtering methods but the most popular is the Gaussian one, whose weighting function is obtained by a Gaussian function (ISO 12181-2, 11562 [5]):

$$
s(x)=\frac{1}{\alpha \lambda_{c o}} \exp \left(-\pi\left(\frac{x}{\alpha \lambda_{c o}}\right)^{2}\right)
$$

where $x$ is the position in relation to the center of the weighting function, $\lambda_{c o}$ is the cut-off wavelength of the profile filter and $\alpha$ is a constant: $\alpha=\sqrt{\ln 2 / \pi}$.

In order to estimate the form deviation, an ideal feature must be associated to the set of measured points using the proper fitting technique. In compliance with ISO 12181-1, the reference circle can be defined based on the four different association methods.

The minimum zone circle (MZCI) computes two concentric circles enclosing the circular profile and having the least radial separation. The least squares circle (LSCI) computes the circle which minimizes the sum of the squares of the local roundness deviations. The minimum circumscribed reference circle (MCCI) is the smallest possible one that can be fitted around the circular profile. On the contrary, the maximum inscribed reference circle (MICI) is the largest possible circle that can be fitted within the circular profile.

Finally, the form deviation can then be assessed as the difference between the maximum and minimum local roundness deviation.

The roundness error $\delta$ is defined as:

$$
\delta=\min \left(\max _{1 \leqslant i \leqslant n} d_{i}-\min _{1 \leqslant i \leqslant n} d_{i}\right)
$$

where $d_{i}$ is the distance of the $i$ th sampled point from the center of the associated circle of coordinates $(a, b)$. It can be expressed as:

$$
d_{i}=\sqrt{\left(x_{i}-a\right)^{2}+\left(y_{i}-b\right)^{2}}
$$

The roundness error $\delta$ is indeed:

$$
\begin{aligned}
\delta= & \sqrt{\left(x_{\max }-a\right)^{2}+\left(y_{\max }-b\right)^{2}} \\
& -\sqrt{\left(x_{\min }-a\right)^{2}+\left(y_{\min }-b\right)^{2}}
\end{aligned}
$$

naming $\left(x_{\max }, y_{\max }\right)$ and $\left(x_{\min }, y_{\min }\right)$ as the coordinates of the points farthest from and nearest to the center of the reference circle.

If all of these operations are completely defined in the geometrical tolerance callout, the specification operator is complete and, according to the GPS duality principle, the perfect verification operator can be directly derived from it. As stated in ISO 17450-2 [6], the perfect verification operator is not implementable in practice since it assumes ideal metrological characteristics of the measurement instrument. The verification operator is named actual verification operator if it faithfully complies with specification procedure and is implemented effectively. In such a case the verification operator is affected by implementation uncertainty only. Verification operations can be simplified, introducing intentional deviations with respect to the corresponding actual specification operator (for instance, a lower number of sampling points), resulting in a simplified verification operator that is affected also by method uncertainty.

In this paper, we performed all of these operations, using virtual geometrical features, through the simulation of their measurement by means of a virtual CMM, in order to know exactly the measurand shape and the distribution of errors occurring at the different phases of measurement and data analysis process.

We used the least squares method, since it is the most popular and widely employed. In fact, it is implemented in most CMM softwares. Moreover, it allows an estimate of the implementation uncertainty, founded on statistical methodologies.

\section{Uncertainty evaluation}

Geometrical specifications should be complete enough to avoid misinterpretations and suitable to guarantee workpiece functional requirements.

For these reasons, GPS language introduces the uncertainty contributions, that affect products at different stages of their lifecycle. These contributions are presented in ISO 17450-2 and they lead to the definition of the workpiece total uncertainty according to the scheme represented in Figure 1.

This uncertainty composition scheme does not represent hierarchical levels, as the uncertainty contributions are defined independently from each other. Total uncertainty takes into account: measurement uncertainty, related to the verification process; specification uncertainty, 


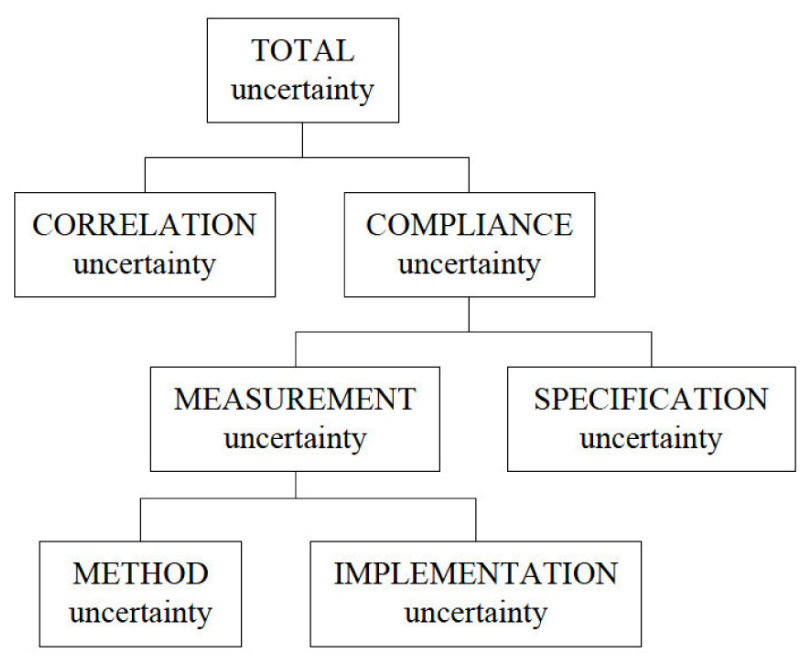

Fig. 1. GPS uncertainty contributions.

which arises when specification operator is not complete; correlation uncertainty, relative to the suitability of the geometrical specification to the workpiece functional requirements. Compliance uncertainty covers both the practical aspects of measurement processes and the quality of specification. Measurement uncertainty collects both method uncertainty, which comes from differences between specification requirements and verification procedures regardless the measurement instrument, and implementation uncertainty, which accounts for deviations of the measurement instrument metrological characteristics.

In this paper, we face the problem of reducing the number of measured points, while maintaining a good level of accuracy in the estimation of implementation uncertainty. This uncertainty contribution is estimated by adopting two methods: the so-called "analytical" one and the wellknown Bootstrapping [7].

The "analytical" approach combines different uncertainty contributions, according to the propagation formula given by the Guide to the Expression of Uncertainty in Measurement [8] and by ISO 14253-2. It is particularly suitable when using the Least Squares association criterion [6], given that the propagation coefficients (partial derivatives) can be directly computed from the analytical model of the associated feature (the perfect circle). In this case the implementation uncertainty can be expressed as:

$$
\begin{aligned}
u_{\delta}^{2}= & \left(\frac{\partial \delta}{\partial a}\right)^{2} \operatorname{Var}(a)+\left(\frac{\partial \delta}{\partial b}\right)^{2} \operatorname{Var}(b)+2 \frac{\partial \delta}{\partial a} \frac{\partial \delta}{\partial b} \operatorname{Cov}(a, b) \\
& +\left(\frac{\partial \delta}{\partial x_{\max }} u_{x_{\max }}\right)^{2}+\left(\frac{\partial \delta}{\partial x_{\min }} u_{x_{\min }}\right)^{2} \\
& +\left(\frac{\partial \delta}{\partial y_{\max }} u_{y_{\max }}\right)^{2}+\left(\frac{\partial \delta}{\partial y_{\min }} u_{y_{\min }}\right)^{2}
\end{aligned}
$$

where $u_{x_{\max }}$ and $u_{y_{\max }}$ are the uncertainties associated to the point $\left(x_{\max }, y_{\max }\right)$ and $u_{x_{\min }}$ and $u_{y_{\min }}$ are the uncertainties associated to the point $\left(x_{\min }, y_{\min }\right)$.

On the contrary, Bootstrapping is a computer-based statistical technique to estimate unknown parameters of a distribution. It is based on the construction of a number of resamples of the observed dataset obtained by random sampling with replacement from the original dataset. In the present work bootstrapping is implemented with a number of equal size resamples of the set of measured points of the initial set. It resembles measurements with different distributions of sampling points and works conveniently also for extreme fit association criteria, e.g. minimum zone [9], where the analytical approach application is not straightforward.

\section{Case study}

Much literature has been dedicated to the identification, analysis and modelization of roundness deviation, particularly of systematic components, which are regarded as signatures of the manufacturing process $[7,9]$. Since the general availability of comprehensive computing facilities and progress in software development make the numerical simulation an attractive alternative option, we performed the comparison introduced in Section 3, using simulated circular profiles, affected by systematic deviations.

Starting from the model proposed by Desta et al. [4], five circular profiles were generated adding to the nominal radius $r_{0}=2.5 \mathrm{~mm}$ a roundness error modeled through a Fourier series (6). The harmonics content of each simulated profile is listed in Table 1.

$$
r(\theta)=r_{0}+\sum_{k=1}^{N}\left(a_{k} \cos (k \theta)+b_{k} \sin (k \theta)\right) \quad 0 \leqslant \theta<2 \pi
$$

Table 1 provides the classification of harmonics, according to their technological meaning: the harmonics up to the 15th generated form errors, those from the 15th to the 100th generated waviness errors, while the harmonics of higher order can be considered as a roughness error contribution.

The actual roundness deviation, in the rightmost column, which accounts for all the harmonics, is the value found by the perfect verification operator defined in the following.

The symbol in the first box of the tolerance callout shown in Figure 2 indicates the type of error that must be controlled (roundness). It has to be lower than the fixed value reported in the second box $(0.1 \mathrm{~mm})$. The third box contains the specification of the filter type (in this case, a Gaussian filter) and its bandwidth: from 1 to 15 undulations per revolution (UPR). The filter bandwidth limits the stylus tip radius values and constrains the number of points to be measured. In fact, the ratio between the measurand diameter $(5 \mathrm{~mm})$ and stylus tip radius $(1 \mathrm{~mm})$ needs to respect the values recommended by ISO 12181-2 (minimum $d / r=5$, where $d$ is the nominal circle diameter and $r$ is stylus tip radius). Moreover, in compliance with the Nyquist Theorem, in order to avoid aliasing, at least seven points per UPR must be sampled.

Finally, the fourth box prescribes the association criterion that has to be used to estimate the associated 
Table 1. Coefficients of the harmonics defining the simulated roundness profiles $(\mu \mathrm{m})$.

\begin{tabular}{|c|c|c|c|c|c|c|c|c|c|c|c|c|c|c|}
\hline \multirow{2}{*}{$\begin{array}{l}\text { Profile } \\
\text { ID }\end{array}$} & & \multicolumn{6}{|c|}{ Form } & \multicolumn{3}{|c|}{ Waviness } & \multicolumn{3}{|c|}{ Roughness } & \multirow{2}{*}{$\begin{array}{c}\text { Actual } \\
\text { roundness }\end{array}$} \\
\hline & $\gamma$ & 2 & 3 & 7 & 10 & 12 & 13 & 20 & 41 & 70 & 300 & 700 & 900 & \\
\hline 1 & $\begin{array}{l}a_{k} \\
b_{k}\end{array}$ & & 30 & 20 & 15 & & & & & $\begin{array}{c}2 \\
1.5\end{array}$ & 3 & 1 & 0.5 & 100.16 \\
\hline 2 & $\begin{array}{l}a_{k} \\
b_{k}\end{array}$ & & $\begin{array}{c}1 \\
55\end{array}$ & & & & & & & $\begin{array}{c}2 \\
1.5\end{array}$ & 3 & 1 & 0.5 & 106.92 \\
\hline 3 & $\begin{array}{l}a_{k} \\
b_{k}\end{array}$ & $\begin{array}{l}10 \\
10\end{array}$ & 40 & & & & & & & $\begin{array}{c}2 \\
1.5 \\
\end{array}$ & 3 & 1 & 0.5 & 101.64 \\
\hline 4 & $\begin{array}{l}a_{k} \\
b_{k}\end{array}$ & & $\begin{array}{c}30 \\
1 \\
\end{array}$ & $\begin{array}{c}20 \\
5 \\
\end{array}$ & & & $\begin{array}{l}5 \\
2 \\
\end{array}$ & & & $\begin{array}{c}2 \\
1.5 \\
\end{array}$ & 3 & 1 & 0.5 & 99.49 \\
\hline 5 & $\begin{array}{l}a_{k} \\
b_{k}\end{array}$ & & 25 & & & 1 & & 25 & 4 & $\begin{array}{c}2 \\
1.5\end{array}$ & 3 & 1 & 0.5 & 66.49 \\
\hline
\end{tabular}

\section{\begin{tabular}{|l|l|ll|l|}
\hline & 0.1 & G & $1-15$ & LSCI \\
\hline
\end{tabular}}

Fig. 2. Complete specification for roundness tolerance.

circle and to evaluate the roundness error (least squares method).

In Figure 3, the five circular profiles (ID = 1, 2, 3, 4, 5) generated using the harmonics content in Table 1 are reported.

The actual verification operator is implemented by simulating measurements with a CMM, characterized by a maximum permissible error (MPE) of $4 \mu \mathrm{m}$. The simulation consists in running a disc with the stylus tip geometry over the virtual circular profile. At each measured point a measurement error is added in the radial direction, by using a Gaussian distribution with null mean and standard deviation equal to $\mathrm{MPE} / 6$.

The implementation uncertainty is estimated with the analytical approach using equation (5) where $u_{x_{\max }}=$ $u_{x_{\min }}=u_{y_{\max }}=u_{y_{\min }}=M P E / 6$ and the variancecovariance components are obtained applying the least squares method to the set of measured points. Bootstrapping is implemented extracting a set of $10^{4}$ Bootstrap samples for each measurement simulation and assessing the roundness error for each sample. We assume that the standard deviation of the $10^{4}$ error evaluations are a good estimation of the LSCI implementation uncertainty.

\section{Results and discussion}

The evaluation of implementation uncertainty is influenced by the nominal roundness error which affects the simulated profiles. Figure 5 shows the nominal roundness error detected in measuring different numbers of equally spaced points by an ideal CMM with $M P E=0$ (differences with respect to a real instrument would be negligible). The sample sizes were selected in order to evaluate the estimate behavior in the case of a number of points lower and higher than the reference value (105 points from specification).
Each considered set of measured points is obtained sampling from a large set of points on the simulated profiles. The implementation uncertainty has been then estimated for each set of sampled points.

The values of implementation uncertainty estimated by using equation (5) are reported in Figure 6; they approximately span from 1 to $30 \mu \mathrm{m}$, depending on the profile. It can be noticed that for three profiles (1,2 and 5) there is no advantage in using more than 30 points (less than $30 \%$ of sample size required by standards). It is wellknown that implementation uncertainty would naturally increase with the reduction of sampling points, following an exponential trend, if the measurand was a calibrated artefact (hence if the measured error was constant). Different trends in implementation uncertainty, as those in Figure 6, depend on the content of roundness deviation actually measured, which is very sensitive to the number of sampled points (see Fig. 5).

When the sample size increases the implementation uncertainty tends to decrease to the natural limit, that is the uncertainty associated with the measurement equipment, and it becomes independent from both the selected profile and the number of sampled points.

The values of implementation uncertainty estimated by using Bootstrapping (Fig. 7) span from 4 to $21 \mu \mathrm{m}$. It increases with low numbers of sampling points, if nominal roundness error is almost constant (see profiles 2, 3 and 4 in Fig. 7).

Nevertheless, there are some peculiar results for the profile 1 and 5. According to the profile 1, the measured error decreases with low numbers of sampling points since most of the form error occurs at frequencies very near to the cut-off one (7 and $10 \mathrm{UPR}$, as reported in Tab. 1). Hence, according to Nyquist theorem these frequencies of form error are naturally overlooked as the sample size decreases. As a consequence bootstrapping a sample with a low detected roundness error gives a low standard deviation of the estimated error, that is a low value of implementation uncertainty. With respect to the profile 5, there is not just a reduction of uncertainty increase when the sample size is reduced, but there is a local maximum of implementation uncertainty with 30 sampling points. A detailed analysis was carried out to investigate this 

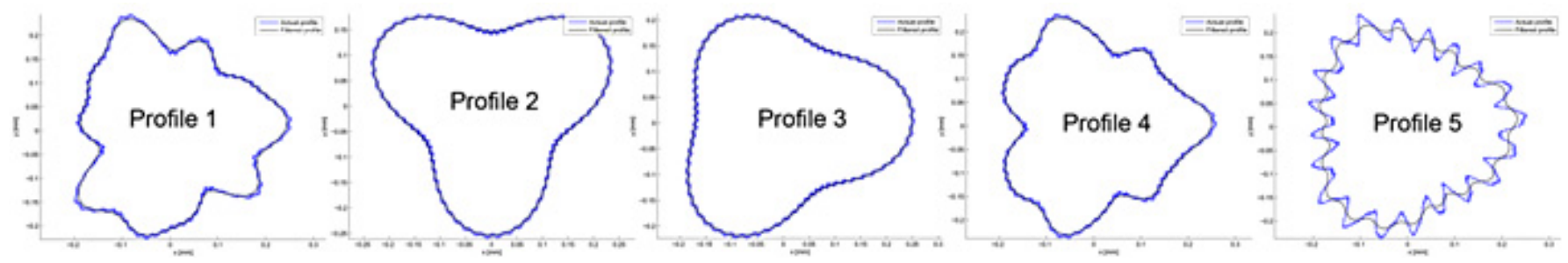

Fig. 3. Roundness profiles, figure out of scale - form error magnified for clarity.

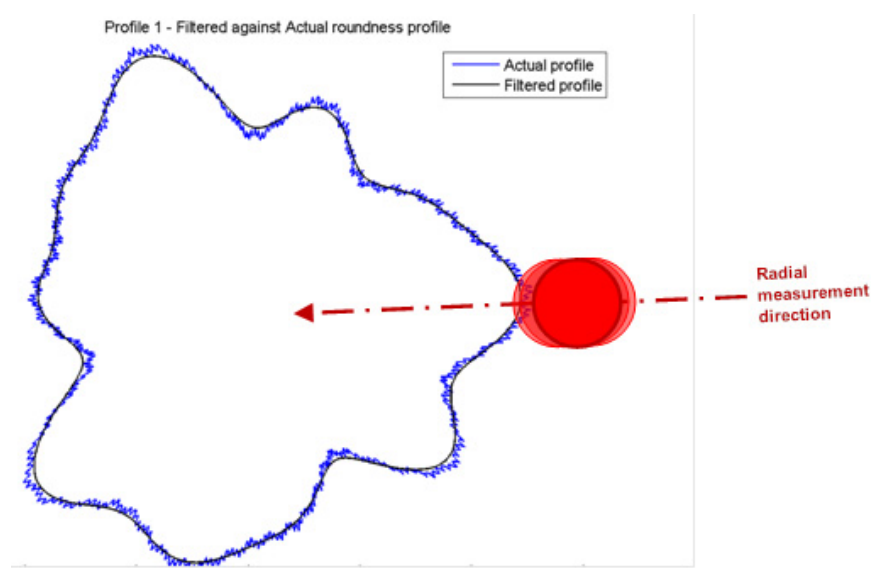

Fig. 4. Measurement simulation, figure out of scale - form error magnified for clarity.

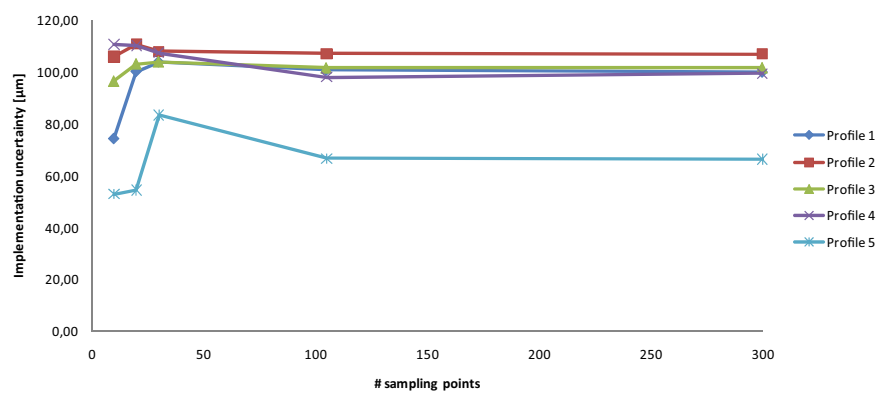

Fig. 5. Nominal roundness error on profiles.

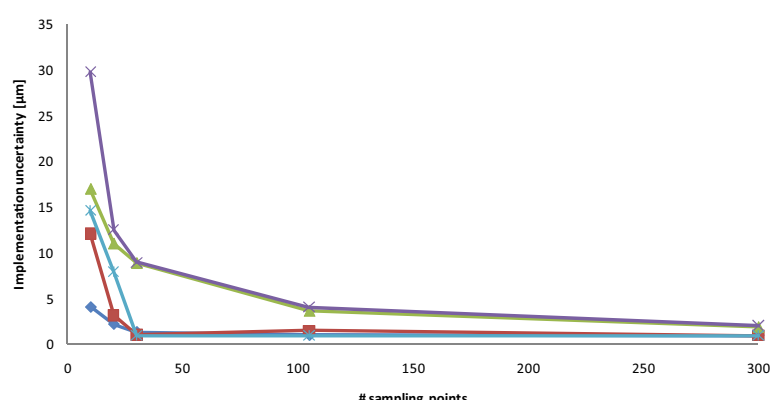

Fig. 6. Implementation uncertainty estimated with "analytical" approach.

behaviour, focusing on values of nominal roundness error and implementation uncertainty in the neighbourhood of 30 points (see Figs. 8 and 9, where circled points are those, respectively, reported in Figs. 5 and 7 ).

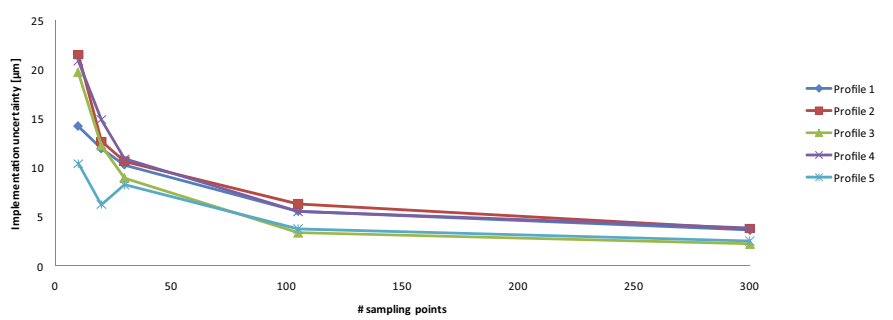

Fig. 7. Implementation uncertainty estimated with Bootstrapping.

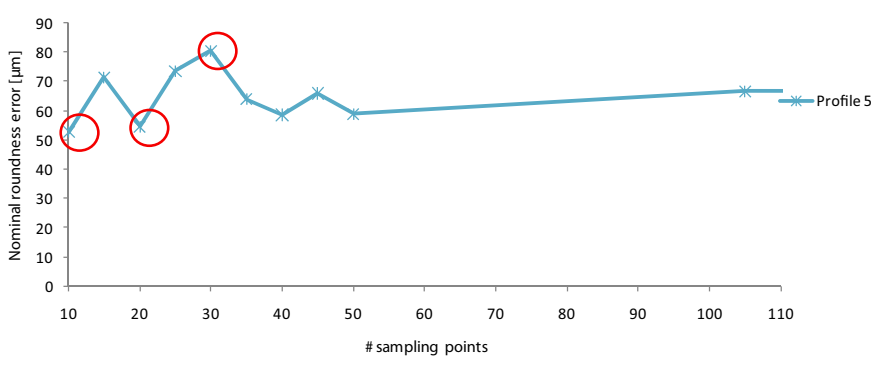

Fig. 8. Detailed analysis of nominal roundness error on profile 5.

These results are mainly due to the interaction between the sampling densities and the profile form, and to the progressive loss of the filter effectiveness below 105 sampling points. The profile 5 is very effective in showing how the reduction of sampling points should be always driven by knowledge of the actual manufacturing signature in order to preserve the effectiveness of the verification operators. If no a priori information about the manufacturing signature (e.g. parameters of finishing operations) is available, and if it is not possible to repeat the measurement changing the location of sampling points to explore different areas of the measurand, the estimated implementation uncertainty is of little use either if it is estimated with the "analytical" approach or by bootstrapping.

In order to account for the repeatability of verification operators and for their coverage of the actual roundness deviation, particularly when these consist of few (less than 100) points, the measurement simulation has been repeated $10^{4}$ times. Particularly, for each simulation, evenly spaced measurement points are sampled starting from a random angle in order to statistically explore all the measurand. It is important to notice that measurement replications are rarely implementable (even with few replications) in industrial practice for the large time 


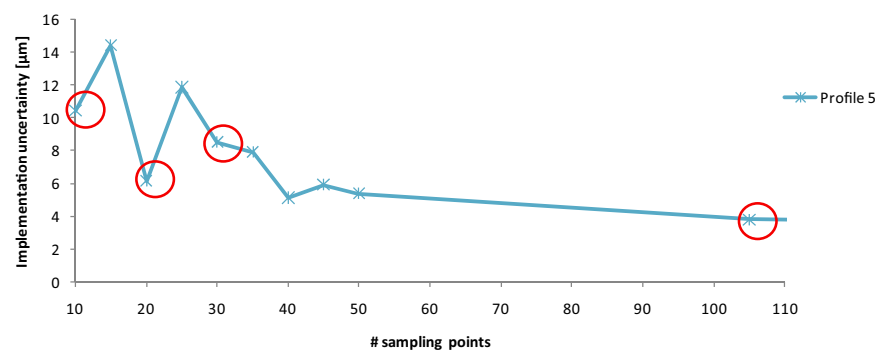

Fig. 9. Detailed analysis of implementation uncertainty for profile 5, with Bootstrapping.

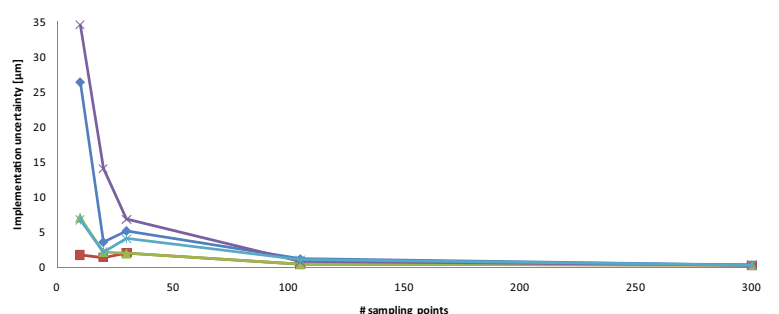

Fig. 10. Implementation uncertainty estimated with measurement replications.

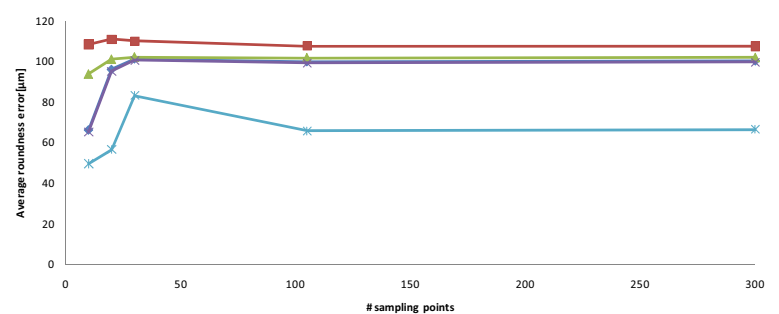

Fig. 11. Average roundness error estimated with measurement replications.

consumption and cost. However, in a simulation context like this one, it provides a valuable statistical reference for the implementation uncertainty. Figures 10 and 11, respectively, resume the replication results in terms of implementation uncertainty and average roundness error. Particularly, implementation uncertainty shown in Figure 10 is estimated as the standard deviation of the roundness values from the $10^{4}$ replications.

Figures 10 and 11 show particular results relative to implementation uncertainty estimation in profiles 1 and 5, confirming what was already noticed by applying the previous two methods.

\section{Conclusions}

In this paper, different methods for implementation uncertainty evaluation were implemented. Particularly, we compared the so called "analytical" approach with Bootstrapping, as these two methods are proposed in the quoted literature.
Given that the study was carried out on simulated data, we could provide a control term of the two different methodologies running several replications of simulated measurements (method rarely implementable in industrial practice, because of its cost in terms of time and money).

The results presented in this paper suggest paying a close attention in the selection of the implementation uncertainty estimation method. If the "analytical" approach seems to be a good tradeoff between the estimation accuracy and the computational costs, it fails when there is a high probability that the workpiece is out of tolerance. In this case Bootstrapping is preferable given that it provides a higher estimation of uncertainty, thus it prevents possible mistakes in accepting non conforming work pieces.

Moreover the acquisition of information about the manufacturing process before setting up the measurement procedure plays an important role. This knowledge together with the selection of a suitable method brings to a correct detection of the real error on the workpiece and an accurate estimation of the implementation uncertainty.

\section{References}

1. V. Srinivasan, Standardizing the specification, verification, and exchange of product geometry: Research, status and trends, Comput. Aided Des. 40, 738-749 (2008)

2. ISO 12181-1, Geometrical product specifications (GPS) Roundness - Part 1: Vocabulary and parameters of roundness (2011)

3. ISO 12181-2, Geometrical product specifications (GPS) Roundness - Part 2: Specification operators (2011)

4. M.T. Desta, H.-Y. Feng, D. OuYang, Characterization of general systematic form errors for circular features, Int. J. Mach. Tools Manufact. 43, 1069-1078 (2003)

5. ISO 11562, Geometrical Product Specifications (GPS) Surface texture: Profile method - Metrological characteristics of phase correct filters (1996)

6. S.F. Changcai Cui, H. Fugui, Research on the uncertainties from different form error evaluation methods by CMM sampling, Int. J. Adv. Manuf. Technol. 43, 136-145 (2009)

7. B. Efron, Bootstrap Methods: Another Look at the Jackknife, Ann. Statist. 7, 1-26 (1979)

8. JCGM 100, Evaluation of Measurement Data - Guide to the Expression of Uncertainty in Measurement (GUM, 2008)

9. S.A. Farooqui, T. Doiron, C. Sahay, Uncertainty analysis of cylindricity measurements using bootstrap method, Measurement 42, 524-531 (2009)

10. ISO 17450-2, Geometrical product specifications (GPS) General concepts - Part 2: Basic tenets, specifications, operators, uncertainties and ambiguities (2012)

11. N. Cho, J. Tu, Roundness modeling of machined parts for tolerance analysis, Precis. Eng. 25, 35-47 (2001)

12. R.P. Henke et al., Methods for evaluation of systematic geometric deviations in machined parts and their relationships to process variables, Precis. Eng. 23, 273-292 (1999) 